\title{
Enhanced Oral Delivery of Docetaxel Using Thiolated Chitosan Nanoparticles: Preparation, In Vitro and In Vivo Studies
}

\author{
Shahrooz Saremi, ${ }^{1,2}$ Rassoul Dinarvand, ${ }^{1,3}$ Abbas Kebriaeezadeh, \\ Seyed Nasser Ostad, ${ }^{3,4}$ and Fatemeh Atyabi ${ }^{1,3}$ \\ ${ }^{1}$ Department of Pharmaceutics, Faculty of Pharmacy, Tehran University of Medical Sciences, Tehran 1417614411, Iran \\ ${ }^{2}$ RéD Department, Osvah Pharmaceutical Co., Tehran, Iran \\ ${ }^{3}$ Nanotechnology Research Centre, Tehran University of Medical Sciences, Tehran 1417614411, Iran \\ ${ }^{4}$ Department of Toxicology and Pharmacology, Faculty of Pharmacy, Tehran University of Medical Sciences, Tehran, Iran
}

Correspondence should be addressed to Fatemeh Atyabi; atyabifa@tums.ac.ir

Received 4 April 2013; Revised 23 June 2013; Accepted 24 June 2013

Academic Editor: Frederic Lagarce

Copyright (C) 2013 Shahrooz Saremi et al. This is an open access article distributed under the Creative Commons Attribution License, which permits unrestricted use, distribution, and reproduction in any medium, provided the original work is properly cited.

\begin{abstract}
The aim of this study was to evaluate a nanoparticulate system with mucoadhesion properties composed of a core of polymethyl methacrylate surrounded by a shell of thiolated chitosan (Ch-GSH-pMMA) for enhancing oral bioavailability of docetaxel (DTX), an anticancer drug. DTX-loaded nanoparticles were prepared by emulsion polymerization method using cerium ammonium nitrate as an initiator. Physicochemical properties of the nanoparticles such as particle size, size distribution, morphology, drug loading, and entrapment efficiency were characterized. The pharmacokinetic study was carried out in vivo using wistar rats. The half-life of DTX-loaded NPs was about 9 times longer than oral DTX used as positive control. The oral bioavailability of DTX was increased to $68.9 \%$ for DTX-loaded nanoparticles compared to $6.5 \%$ for positive control. The nanoparticles showed stronger effect on the reduction of the transepithelial electrical resistance (TEER) of Caco-2 cell monolayer by opening the tight junctions. According to apparent permeability coefficient $\left(P_{\text {app }}\right)$ results, the DTX-loaded NPs showed more specific permeation across the Caco-2 cell monolayer in comparison to the DTX. In conclusion, the nanoparticles prepared in this study showed promising results for the development of an oral drug delivery system for anticancer drugs.
\end{abstract}

\section{Introduction}

In recent years, many works have been focused on the development of oral chemotherapy systems. Docetaxel (DTX) is regarded as one of the most effective drugs used in chemotherapy. DTX is a semisynthetic taxoid extract from Taxus baccata (European yew tree) and is used as antineoplastic agent against breast, ovarian, lung, head, and neck cancers [1-3]. The mechanism of action of DTX like other taxanes is inhibiting microtubule depolymerization $[4,5]$. Because of stronger binding of DTX to tubulin it shows about 2-4 times more cytotoxicity effects on tumor cells than that of paclitaxel [6]. However DTX like other taxans has a poor oral absorption from gastrointestinal (GI) tract [7] and the only dosage form of DTX in the market is an injection dosage form (Taxotere). The main reasons for the poor oral bioavailability of DTX are related to low solubility of DTX in water, its high affinity to the multidrug efflux pump Pglycoprotein (P-gp), and hepatic first pass metabolism $[8,9]$. Due to the poor solubility of DTX in water, it has been formulated as a solution using high amount of Tween 80 in ethanol $(50: 50 \mathrm{v} / \mathrm{v})$. High concentration of solubilizers in its formulation causes toxic effects and allergic reactions [10]. Various methods have been suggested to overcome these problems such as applying a P-gp/P450 inhibitor such as cyclosporine A [11, 12], formulated as liposomes [13, 14], emulsions $[15,16]$, polymeric nanoparticles [17-21], and conjugation of DTX with water soluble polymers $[22,23]$.

Preparing surface modified polymeric nanoparticle may also be regarded as an effective mode of overcoming these problems. Nanoparticles, due to their unique properties and surface characteristics, can protect the drug from P-gp, 
cytochrome P-450, and from the destructive factors in the GI tract and can increase the permeability of drugs through the gastrointestinal barrier [24, 25]. Dong and Feng [19] developed nanoparticles using biodegradable polymers and showed that these polymeric nanoparticles can significantly increase oral bioavailability of DTX in rat. In another study, Peltier et al. [26] reported an increase in transport through the intestinal barrier and oral bioavailability of paclitaxel by lipid nanoparticles. Agüeros et al. [27] showed that, when paclitaxel was encapsulated in a complex of cyclodextrins and poly (anhydride) nanoparticles, its bioavailability was significantly increased. These reports confirm that nanoparticulate systems with unique properties can increase the transport of poorly water-soluble compounds across the GI barrier. In this study we investigated the capacity of prepared thiolated nanoparticles based on thiolated chitosan to improve the oral bioavailability of DTX as a model anticancer drug with poor oral absorption. Roldo et al. [28] showed that the mucoadhesive properties of chitosan was enhanced 140 -fold due to the immobilization of thiol groups on the polymer. Formation of disulfide bonds between the thiolated polymer and cysteine-rich subdomains of the mucus gel layer is responsible for this improvement [29]. There are many reports on the application of thiolated chitosan for enhancing permeability, mucoadhesivity and intestinal absorption of active agents [30-33].

Recently, we reported that DTX and paclitaxel could be easily entrapped in thiolated chitosan-pMMA nanoparticles $[34,35]$. It was shown that drug-loaded NPs increased the cytotoxicity of DTX and transportation of DTX across the jejunum of rats was facilitated in ex vivo study. TEER value of Caco-2 cell monolayer was also measured to evaluate the influence of the thiolated nanoparticles on the quality of intestinal tight junctions in male Wistar rats.

\section{Materials and Methods}

2.1. Materials. Docetaxel was obtained from Cipla (Mumbai, India), Taxotere, an injectable commercially available formulation of DTX, was from Sanofi-Aventis (France), and Chitosan (ChitoClear) with molecular weight of 20 and $50 \mathrm{kDa}$ and degree of deacetylation of about $89 \%$ was purchased from Primex (Karmoy, Norway). L-Glutathione reduced form (GSH), 1-ethyl-3-(3-dimethylaminopropyl) carbodiimide hydrochloride (EDC), N-hydroxysuccinimide (NHS), methyl methacrylate (MMA), ammonium cerium nitrate, sodium nitrite, hydrochloric acid, glacial acetic acid, sodium hydroxide, and potassium hydrogen phosphate were all purchased from Merck (Darmstadt, Germany). Ellman's reagents, 5, 50-dithiobis (2-nitro benzoic acid), were obtained from Sigma (St. Louis, MO, USA). Caco2 cell lines were obtained from Pasteur Institute (Tehran, Iran). 3-(4,5-Dimethylthiazol-2-yl)-2,5-diphenyl tetrazolium bromide) (MTT) and all of the cell culture mediums were purchased from Sigma-Aldrich (St. Louis, MO, USA). All other chemicals were of analytical grade.
2.2. Preparation of DTX-Loaded Nanoparticle. Thiolated chitosan was prepared with covalent attachment of reduced glutathione to chitosan in the presence of EDC and NHS according to method described in our previous study [36]. The DTX-loaded nanoparticles were prepared by using a modified radical polymerization method [37]. Conjugated chitosan $(37.5 \mathrm{mg}$ ) was dissolved in $4 \mathrm{~mL}$ nitric acid $(0.2 \mathrm{M})$ in a two-necked flask under gentle stirring and nitrogen bubbling at $40^{\circ} \mathrm{C}$. After $10 \mathrm{~min}$, under vigorous magnetic stirring, solution of $0.08 \mathrm{M}$ cerium IV ammonium nitrate $(\mathrm{CAN})$ in $0.2 \mathrm{M}$ nitric acid was added to obtain a $5 \mathrm{~mL}$ solution. DTX was dissolved in a $0.5 \mathrm{~mL}$ of methanol under stirring. Then $0.25 \mathrm{~mL}$ MMA was added to obtain a clear solution. The added amount of DTX was $10.90 \mathrm{mg}$ or $4 \%$ $(\mathrm{w} / \mathrm{w})$ based on total weight of polymers (MMA and thiolated chitosan). Nitrogen bubbling was kept for additional $10 \mathrm{~min}$. The reaction was allowed to continue at $40^{\circ} \mathrm{C}$ under gentle stirring for $40 \mathrm{~min}$. The reaction was left to reach room temperature, and the $\mathrm{pH}$ of obtained suspension adjusted to 4.5 by addition of sodium hydroxide $(1 \mathrm{~N})$ dropwise. Then nanoparticles suspension was purified by dialyzing against acetic acid solution, used for the removal of the remained methacrylic acid monomers, $(1 \mathrm{~L}, 16 \mu \mathrm{mol} / \mathrm{L})$ in demineralized water for 90 min twice and once overnight using Sigma dialysis tubes $\mathrm{Mw}$ cutoff of $12 \mathrm{kDa}$. The frozen samples were lyophilized at $-50^{\circ} \mathrm{C}$ and $0.01 \mathrm{mbar}$ and stored at $4^{\circ} \mathrm{C}$ until further use.

2.3. Characterization of Nanoparticles. The mean diameter and size distribution of nanoparticles were determined by dynamic light scattering using Zetasizer (Nano-ZS, Malvern, Worcestershire, UK) at wavelength of $633 \mathrm{~nm}$ at $25^{\circ} \mathrm{C}$ with an angle detection of $90^{\circ}$. The samples were diluted in acetic acid $(16 \mu \mathrm{mol} / \mathrm{L})$ in deionized water, and three subsequent measurements were determined for each sample, and the result was expressed as mean size \pm S.D. The zeta potential measurements were performed by laser Doppler electrophoresis using Zetasizer (Nano-ZS, Malvern, Worcestershire, UK). In order to maintain a constant ionic strength, the samples were diluted (1:50 v/v) in $\mathrm{NaCl} 1 \mathrm{mM}$ (pH_6.5) [38]. Each sample was repeatedly measured three times.

The surface morphology of nanoparticles was evaluated by using a scanning electron microscope (XL 30, Philips, Eindhoven, the Netherlands). Nanoparticle suspensions were successively diluted in deionized water to $1 / 50(\mathrm{v} / \mathrm{v})$. The dilutions were spread on an aluminum disc and dried at room temperature before the analysis. The dried nanoparticles were then coated with a thin layer of gold metal using a sputter coater (SCD 005, Bal-Tec, Switzerland).

2.4. Drug Loading and Entrapment Efficiency. The entrapment efficiency (EE) of the process was determined indirectly upon separation of the drug-loaded NPs (after dialysis) by ultracentrifugation at $25,000 \mathrm{rpm}, 8^{\circ} \mathrm{C}$ for 30 minutes from the aqueous medium containing free DTX. The amount of free DTX in the supernatant was measured using HPLC. Isocratic reversed-phase HPLC was performed using a Knauer 
HPLC system (Knauer, Berlin, Germany) with a $5 \mu$ Bondapak C18 column (Waters, Milford, MA, USA). The mobile phase consisted of $75: 25(\mathrm{v} / \mathrm{v})$ methanol/water and was delivered at a flow rate of $1.0 \mathrm{~mL} / \mathrm{min}$. Eluted compounds were detected at $227 \mathrm{~nm}$ using a Spectra100 UV-Vis detector. The standard curve was found to be linear in the concentration range $0.5 \mu \mathrm{g} / \mathrm{mL}-50 \mu \mathrm{g} / \mathrm{mL}$ with $R^{2}=0.9999$.

The EE of DTX NPs was calculated as the ratio of DTX loaded into the NPs with respect to the total amount of DTX used for preparation of the original mixture as follows:

$$
\mathrm{EE}(\%)=\frac{(\mathrm{DTXt}-\mathrm{DTXf})}{\mathrm{DTXt}} \times 100
$$

where DTXt is the total amount of DTX used for preparation of the original mixture and DTXf is the free DTX amount recovered in the supernatant. All samples were measured in triplicate. Drug loading was calculated as follows [39]:

$$
\operatorname{DL}(\%)=\left(\frac{\text { Weight of loaded drug }}{\text { Weight of NPs }}\right) \times 100 \text {. }
$$

2.5. In Vitro Drug Release Study. Drug release from DTXloaded nanoparticles was studied by incubating the nanoparticles in phosphate buffer solutions (PBS), at $\mathrm{pH} 7.4$, at $37^{\circ} \mathrm{C}$. Two $\mathrm{mg}$ of nanoparticles were dispersed in $5 \mathrm{~mL}$ of release medium (PBS of $\mathrm{pH} 7.4$ containing $0.1 \% \mathrm{w} / \mathrm{v}$ Tween 80 ) in a dialysis tube (Sigma dialysis tubes $\mathrm{Mw}$ cutoff $12 \mathrm{kDa}$ ), and the closed dialysis bag immersed in $20 \mathrm{~mL}$ release medium in a centrifuge tube. Tween 80 was used to increase the solubility of DTX in the buffer solution to maintain sink condition. The tube was placed in a shaker bath at $37^{\circ} \mathrm{C}$ and shaken horizontally at 100 cycles/min. At given time intervals, $15 \mathrm{~mL}$ of samples were withdrawn and replaced with the same volume of fresh medium. The samples were filtered through a $0.22 \mu \mathrm{m}$ filter and were analyzed for the amount of DTX using HPLC.

2.6. Caco-2 Cell Culture Study. Caco-2 cells, with a passage number 40-45, were cultured on polycarbonate membrane filters (pore size $0.4 \mu \mathrm{m}$, area $0.47 \mathrm{~cm}^{2}$ ) in 24 -well plates (Nunc, Roskilde, Denmark) at a seeding density of $4 \times$ $10^{5}$ cells $/ \mathrm{cm}^{2}$. The RPMI $1640(50 \% \mathrm{v} / \mathrm{v})$, Dulbeccos modified Eagle's medium (35\% v/v DMEM, Sigma, pH 7.4), with $4500 \mathrm{mg} / \mathrm{L}$ glucose and 15\% fetal bovine serum (FBS) containing $1 \%$ penicillin-streptomycin was used as medium for cell culture. The culture medium was added to both the apical $(300 \mu \mathrm{L})$ and basolateral $(700 \mu \mathrm{L})$ of filter insert and was changed every other day for the first 10 days and every day thereafter until 21 days. The cells were left at $37^{\circ} \mathrm{C}$ in an incubator under atmosphere of $95 \%$ air and $5 \% \mathrm{CO}_{2}$ at $90 \%$ relative humidity. One hour before the experiments, the medium was changed to the transport buffer containing: Hank's balanced salt solution (HBSS) buffered with $30 \mathrm{mM}$ n-(2-hydroxyethyl) piperazine-n-(2ethanesulfonic acid) (HEPES) at pH 5.5 and the cells were allowed to equilibrate for $1 \mathrm{~h}$.
2.7. Determination of the Transepithelial Electrical Resistance (TEER). The integrity of cell monolayer on the filters was examined by measuring the transepithelial electrical resistance (TEER) using an EVOM2 instrument (World precision Instruments, Sarasota, FL) connected to a pair of chopstick electrodes. TEER test was carried out to examine the ability of DTX and DTX-loaded NPs on the opening of the tight junctions at predetermined time intervals of $0,0.5,1,2,3,4$, and $24 \mathrm{~h}$. The experiments were done in triplicate.

2.8. Permeation Study. Permeation of samples was determined as described by Sadeghi et al. [40] with some modifications. Transport of different dispersions of free DTX, Taxotere, commercially available formulation of injectable docetaxel (F-DTX), and Ch20-GSH-DTX and Ch50-GSHDTX NPs was studied from the apical to basolateral direction on Caco- 2 cells. The test solutions were produced by diluting with transition buffer (HEPES-HPSS) at $2 \mu \mathrm{M}$ DTX concentrations. The upper chamber (apical side) was filled with $300 \mu \mathrm{L}$ of the different test solutions and the lower chamber (basolateral side) was filled with $700 \mu \mathrm{L}$ of the growth medium followed by incubation at $37^{\circ} \mathrm{C}$ with $5 \%$ $\mathrm{CO}_{2} / 95 \%$ air. At predetermined time of $30,60,120$, and $240 \mathrm{~min} 300 \mu \mathrm{L}$ samples were withdrawn from the basolateral side of filter and replaced with equal volumes of fresh HBSSHEPES. The samples were analyzed for the DTX content using the HPLC method. After four hours and completion of the permeability studies, the samples were carefully removed from the apical part and the cell monolayer was rinsed with HBSS-HEPES, and the medium was then replaced with fresh culture medium. The monolayer was incubated for $24 \mathrm{~h}$ at $37^{\circ} \mathrm{C}$ in regular cell culture conditions. The TEER was monitored during the experiment and at $24 \mathrm{~h}$. Results were corrected for dilution and expressed as cumulative transport with time. All the experiments were done in triplicate.

Apparent permeability coefficients $\left(P_{\text {app }}\right)$ were calculated using the following equation:

$$
P_{\text {app }}=\frac{Q}{A C_{0} t},
$$

where $P_{\text {app }}$ is the apparent permeability coefficient $(\mathrm{cm} / \mathrm{s})$, $Q$ is the total amount permeated throughout the incubation time $(\mu \mathrm{g}), A$ is the diffusion area of the monolayer $\left(\mathrm{cm}^{2}\right)$, $C_{0}$ is the initial concentration of the DTX in the apical part $\left(\mu \mathrm{g} / \mathrm{cm}^{3}\right)$, and $t$ is the total time of the experiments.

2.9. In Vivo Study. Male wistar rats of $250-280 \mathrm{~g}$ and $10-$ 12 weeks old (provided by Pasteur Institute of Iran) were kept at temperature of $25 \pm 2^{\circ} \mathrm{C}$ and relative humidity of 50$60 \%$ under natural light/dark conditions for one week before experiments. The animals were distributed into three groups. Group 1 received an i.v. injection of F-DTX $(n=5)$. Groups 2 and 3, used for oral administration of DTX, were allowed to fast and unlimited for water accessibility for $12 \mathrm{~h}$ followed by receiving an oral delivery of F-DTX and DTX-loaded NPs $(n=5)$, respectively. The study protocol was approved by the Institutional Review Board of Pharmaceutical Research Centre of Tehran University of Medical Sciences. 
TABLE 1: Properties and characteristics of the Ch20- and Ch50-DTX-loaded NPs.

\begin{tabular}{|c|c|c|c|c|c|c|}
\hline Formulation & DTX (mg) & Size $(\mathrm{nm})$ & PDI & Zeta $(\mathrm{mV})$ & DL (\%) & $\mathrm{EE}(\%)$ \\
\hline Ch20-GSH-DTX 4\% & 10.90 & $198 \pm 8.5$ & 0.12 & $+31.6 \pm 1.5$ & 18.39 & 93.56 \\
\hline Ch50-GSH-DTX 4\% & 10.90 & $262 \pm 7.0$ & 0.23 & $+30.6 \pm 4.4$ & 18.22 & 89.22 \\
\hline
\end{tabular}

The NP formulation was dispersed in, and F-DTX was diluted with saline and was orally administered at the same DTX dose of $10 \mathrm{mg} / \mathrm{kg}$ body weight. For Groups 1 and 2, blood samples were collected at $0,0.5,1,2,3,5,8,12,24$, and $48 \mathrm{~h}$. For Group 3, blood samples were collected at 0 , $0.5,1,2,3,5,8,12,24,48,72,120,168,196,216,240,360$, and $480 \mathrm{~h}$ after administration. Plasma samples were harvested by centrifugation at $14,000 \mathrm{rpm}$ for $15 \mathrm{~min}$ and stored at $-40^{\circ} \mathrm{C}$ for HPLC analysis.

2.10. Drug Loading and Release Measurements. HPLC method as reported previously [34] was used for the analysis of DTX for the drug content, transport, and in vitro release studies. Samples were directly injected $(20 \mu \mathrm{L})$ into the HPLC system without further treatment, while plasma samples were extracted with chloroform and dichloromethane before injection. Briefly, $500 \mu \mathrm{L}$ of plasma was spiked with $200 \mu \mathrm{L}$ of phosphate buffer ( $\mathrm{pH} 6.5)$ and $25 \mu \mathrm{L}$ of paclitaxel $(20 \mu \mathrm{g} / \mathrm{mL}$ in ethanol) as the internal standard. DTX was extracted with $5 \mathrm{~mL}$ chloroform and $700 \mu \mathrm{L}$ dichloromethane by vigorous mixing for $1 \mathrm{~min}$. After centrifugation at $3500 \mathrm{rpm}$ for $15 \mathrm{~min}$, the organic phase was collected. The organic phase was dried under nitrogen gas stream at $40^{\circ} \mathrm{C}$. The residue was then dissolved with $70 \mu \mathrm{L}$ of mobile phase and mixed for $5 \mathrm{~min}$. The solution was centrifuged for $2 \mathrm{~min}$ at $3000 \mathrm{rpm}$, and $20 \mu \mathrm{L}$ of the supernatant was injected into the HPLC system (Knauer, Berlin, Germany) using a spectra $100 \mathrm{UV}$ Vis detector.

For plasma samples a Nucleodur C18 Gravity HPLC packed column $(4.6 \mathrm{~mm} \times 250 \mathrm{~mm}, 5 \mu \mathrm{m}$, Macherey-Nagel, Germany) was used at room temperature. The mobile phase (phosphate buffer ( $\mathrm{pH}$ 6.0): methanol (70:30 v/v)) flowed at rate of $1.3 \mathrm{~mL} / \mathrm{min}$. Eluted compounds were detected at $227 \mathrm{~nm}$. The total run time was $25 \mathrm{~min}$.

2.11. Statistical Analysis. Data are reported as mean \pm standard deviation from 3 independent experiments. Statistical significance between mean values was calculated using independent sample $t$-test and one-way analysis of variance (ANOVA). Probability values $<0.05$ were considered significant.

\section{Results and Discussions}

3.1. Preparation and Characterization of DTX-LoadedNanoparticles. Table 1 shows the particle size, polydispersity index (PDI), and zeta potential of NPs prepared using thiolated chitosan having two different molecular weight of 20 and $50 \mathrm{kDa}$ (Ch20-GSH, Ch50-GSH). As can be seen the mean diameters of the NPs were $198 \mathrm{~nm}$ for Ch20 and $262 \mathrm{~nm}$ for Ch50. As expected nanoparticles prepared

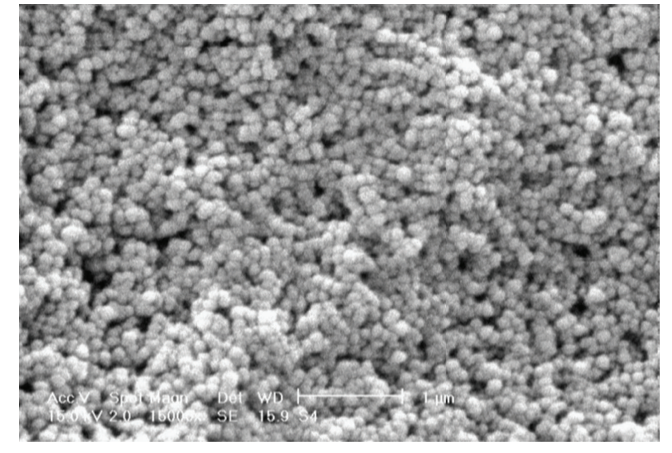

FIGURE 1: Scanning electron micrograph of DTX-loaded nanoparticles (magnification of $150,000 x$ ).

with higher molecular weight chitosan were bigger in size compared with those prepared with smaller molecular weight chitosan. This may be due to the higher viscosity of polymeric droplets of higher molecular weight chitosan compared with those of smaller molecular weight chitosan. The results were very similar to the results obtained by Bernkop-Schnürch et al. [39]. The PDI values of NPs were 0.12 and 0.20 for Ch20 and Ch50, respectively, indicating a more homogenous size distribution for nanoparticles prepared by lower molecular weight chitosan. The zeta potential of the NPs was positive due to the presence of amino groups of chitosan around the core of pMMA. This positive zeta potential and the existing of thiol groups on the surface of particles are favorable for making strong electrostatic and disulfide bonds with negatively charged mucosa which can prevent elimination of the nanoparticles through the alimentary canal [41].

Figure 1 shows the SEM image of the NPs that are spherical and uniform.

3.2. Entrapment Efficiency and Drug Loading of NPs. Table 1 shows the EE and DL of the NPs. As can be seen in this table, the EE of Ch20-GSH and Ch50-GSH NPs is $93.5 \%$ and $89.2 \%$, respectively. This high EE might be due to the tendency of DTX as a hydrophobic molecule to enter the hydrophobic core (pMMA) of the NPs. Ideally, a successful nanoparticulate system should have a high DL capacity. The DL of Ch20GSH and Ch50-GSH NPs was $18.4 \%$ and $18.2 \%$, respectively. A high DL capacity was expected, due to the hydrophobicity property of DTX.

3.3. In Vitro Drug Release Study. Drug release study showed a sustained release profile as up to $80 \%$ of its drug content released during 10 days from $\mathrm{Ch} 20$ and about $70 \%$ from Ch50 NPs in phosphate buffer solution with $\mathrm{pH}$ of 7.4 (Figure 2). The hydrophobic core of nanoparticles acts as a barrier 


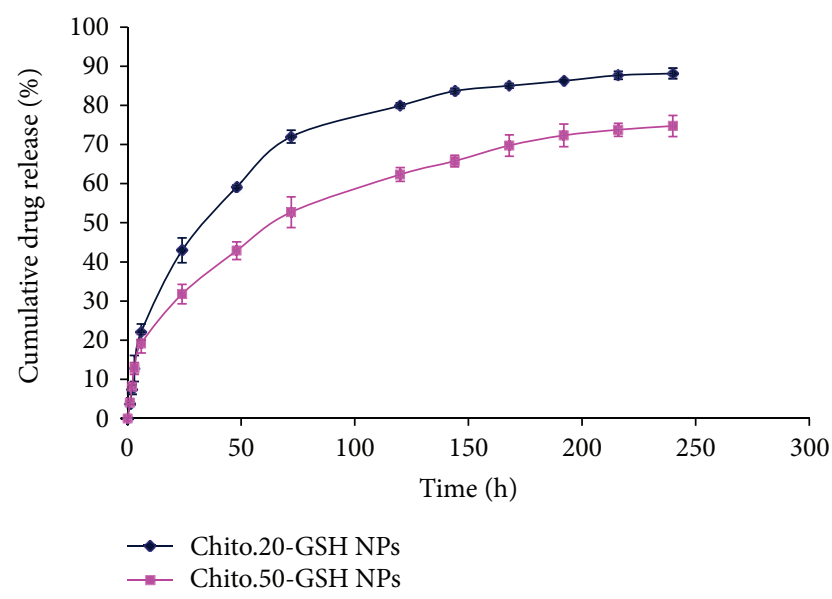

FIgURE 2: In vitro drug release profile of the Ch20-GSH ( $\downarrow)$ and Ch50-GSH ( $)$ NPs. Experiments were carried out in triplicate $(n=$ 3).

against the diffusion of entrapped DTX from the polymeric matrix into the aqueous solution resulting in a slow release of drugs, desirable for thiolated nanoparticles. When NPs were created with Ch50, it was shown that they are dispersed in buffer, but some aggregation and formation of a mass of large particles may be seen. Difference between drug releases behavior of two nanoparticles prepared with chitosan with two different molecular weights might be related to the size of them. NPs prepared with higher molecular weight have bigger size in medium and form a more viscose layer around the particles after hydration with water.

3.4. Effect of Nanoparticle Suspension on TEER of Caco-2 Cell Monolayer. The reversible effects of nanoparticles of thiolated chitosan on barrier properties and opening the intestinal tight junctions were studied by measuring the transepithelial electrical resistance (TEER) values across the Caco-2 cells. The results are presented as the percentage of the initial values at $t=0 \mathrm{~min}$ and are shown in Figure 3. As can be seen, the effect of nanoparticles on opening the tight junction is higher than that for free DTX or F-DTX. After four hours the quantity of opening tight junction with nanoparticles is about $80 \%$ for Ch20 and $88 \%$ for Ch50 of the initial value versus about $92 \%$ and $97 \%$ for F-DTX and free DTX, respectively. One of the possible mechanisms for uptake of nanoparticles via the intestinal tract is paracellular transport that is done through epithelial cells. In many studies it was demonstrated that nanoparticles based on chitosan are able to open tight junction and transport across the cell monolayer [40, 42, 43]. Chitosan derivatives can disrupt epithelial cell tight junctions and decrease the TEER value by two pathway: (1) interaction of their positive surface charge with the anionic components of the glycoprotein on the surface of the epithelial cells $[25,44]$ and (2) translocation of tight junction proteins from the plasma membrane where they are available to form tight junctions membrane to the cytoskeleton [45]. Nanoparticles prepared from smaller molecular weight chitosan (Ch-20) reduced the TEER value

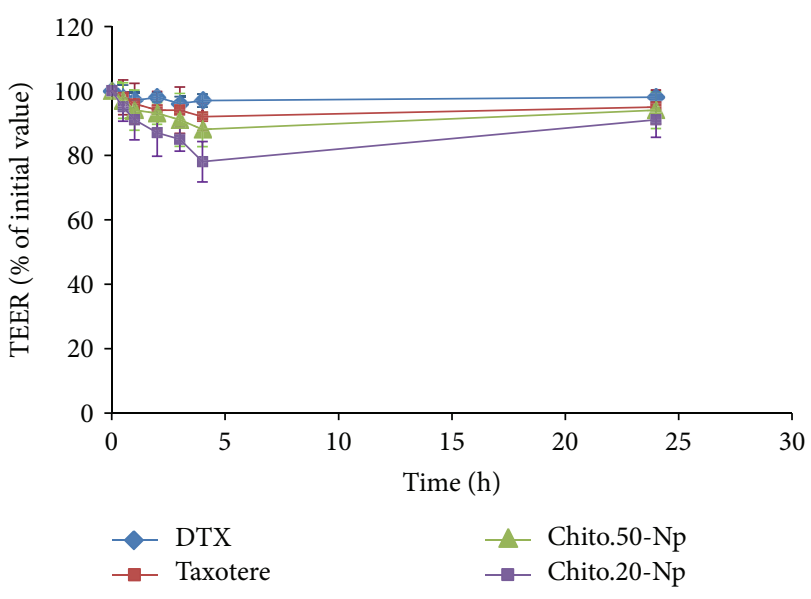

FIGURE 3: Effects of DTX and DTX-loaded nanoparticles on TEER of Caco- 2 cell monolayer during the experiment and $24 \mathrm{~h}$ after rinsing the monolayer with HBSS-HEPES and applying culture medium on the monolayer. Data are expressed as means \pm SD of three experiments.

TABLE 2: Apparent permeability $\left(P_{\text {app }}\right)$ of different samples of DTX: free DTX, F-DTX, and DTX-loaded NPs ( $n=3$; data are showed as mean $\pm \mathrm{SD}$ ); the difference $P<0.05$ is considered as significant.

\begin{tabular}{lc}
\hline Test compound & Average $P_{\text {app }}{ }^{*}\left(\times 10^{-6} \mathrm{~cm} / \mathrm{s}\right)$ \\
\hline DTX & $0.08 \pm 0.14$ \\
F-DTX & $0.38 \pm 0.05$ \\
Ch50-GSH-DTX NPs & $2.14 \pm 0.22$ \\
Ch20-GSH-DTX NPs & $2.43 \pm 0.38$ \\
\hline
\end{tabular}

${ }^{*} P_{\text {app }}$ : apparent permeability.

more substantially than higher molecular weight chitosan nanoparticles (Ch-50). TEER value of F-DTX was shown to be close to Ch-50 and much lower than free DTX. The reason for this observation may be related to the Tween 80 content of F-DTX. It has been shown that nonionic surfactants such as Tween 80 in a large dose are able to enhance the permeability of Caco-2 cell monolayer [46] and decrease the TEER value. F-DTX has a large volume of Tween 80 and can increase the permeability more than free DTX. The $P_{\text {app }}$ values of the DTX in different formulations are shown in Table 2. The $P_{\text {app }}$ value of DTX from Ch20-GSH, Ch50-GSH, F-DTX, and free DTX was $2.43,2.14,0.38$ and 0.08 , respectively. It showed that the apparent permeability values of nanoparticles were significantly higher than those from free DTX and F-DTX.

3.5. In Vivo Pharmacokinetics. Figure 4 shows the mean plasma concentration of DTX when administrated orally using F-DTX and DTX-loaded NPs compared to injected F-DTX at the same concentration $(10 \mathrm{mg} / \mathrm{kg})$ in Wistar rat animals $(n=5)$. Plasma level of DTX was measurable up to $216 \mathrm{~h}$ for NPs (p.o.) and $24 \mathrm{~h}$ for F-DTX when administered orally or intravenously. The most important pharmacokinetic parameters including $C_{\max }, T_{\max }, T_{1 / 2}, \mathrm{AUC}_{0-\infty}$, and MRT are summarized in Table 3 . It can be seen that after intravenous administration of DTX, the drug plasma level reached 
TABLE 3: Pharmacokinetics parameters in rats after i.v. administration of F-DTX and oral administration of F-DTX and Ch20-GSH-DTX $\mathrm{NPs}$ at the same $10 \mathrm{mg} / \mathrm{kg}$ drug dose.

\begin{tabular}{lccc}
\hline PK Parameters & F-DTX (IV) & F-DTX (oral) & Ch20-GSH NPs (oral) \\
\hline$T_{\max }(\mathrm{h})$ & 0.5 & 2 & 5 \\
$C_{\max }(\mathrm{ng} / \mathrm{mL})$ & $14,744 \pm 2,354$ & $456 \pm 54.1$ & $341 \pm 47.5$ \\
$\mathrm{AUC}_{0-\infty}(\mathrm{h} \cdot \mathrm{ng} / \mathrm{mL})$ & $65,245 \pm 4,545$ & $4,243 \pm 207$ & $44,998 \pm 3,534$ \\
$T_{1 / 2}(\mathrm{~h})$ & $2.7 \pm 0.6$ & $11.7 \pm 1.45$ & $102.5 \pm 12.6$ \\
MRT $(\mathrm{h})$ & $3.2 \pm 0.3$ & $15.7 \pm 1.6$ & $144.0 \pm 10.7$ \\
Absolute bioavailability & - & $6.5 \%$ & - \\
Relative bioavailability & - & - & $68.9 \%$ \\
\hline
\end{tabular}

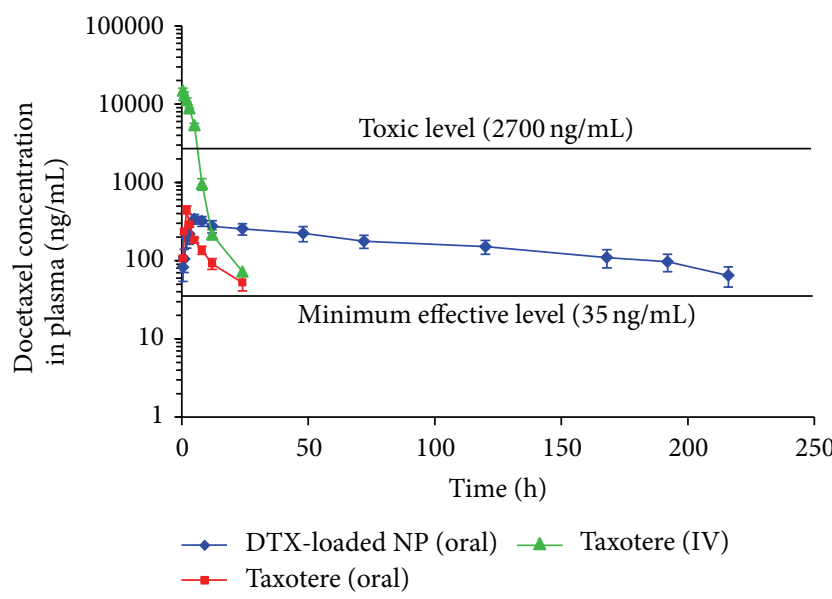

Figure 4: Plasma concentration-time profiles of docetaxel after bolus intravenous injection of F-DTX, oral administration of F-DTX or DTX-loaded NPs (equivalent to $10 \mathrm{mg} / \mathrm{kg}$ as docetaxel) to rats. Each data point represents the mean \pm SD of five determinations.

to extremely high concentration value $(14,744 \mathrm{ng} / \mathrm{mL})$ above the maximum therapeutic level [47] which may cause serious side effects. Instead, oral F-DTX and oral NP formulation showed lower maximum drug concentrations that are in the therapeutic window ( $456 \mathrm{ng} / \mathrm{mL}$ and $341 \mathrm{ng} / \mathrm{mL}$, resp.). As can be seen, drug half-life $\left(T_{1 / 2}\right)$ for oral administration of NPs was $102.5 \mathrm{~h}$, that is, about 9-fold more than F-DTX when given orally. This may be due to the mucoadhesion of NPs that prolong their residence at the site of absorption. As expected the $T_{\max }$ was increased to $5 \mathrm{~h}$ for Ch-GSH NPs, 2.5-fold of that for oral administration of F-DTX. Also the data illustrated that bioavailability of DTX formulated in Ch-GSH NPs is $68.9 \%$ which is about 10 -fold more than that for oral bioavailability of F-DTX (6.5\%). This significant increase in the oral bioavailability of DTX in the NPs formulation could be related to mucoadhesion properties, P-gp efflux inhibition, and permeability enhancing effects of thiolated chitosan. Given the prolonged plasma level of docetaxel when nanoparticles are given orally, the absorption of nanoparticles is a real possibility. Another explanation of this higher plasma level of docetaxel for nanoparticles may be related to the mucoadhesion of nanoparticles. In addition to that, it is well established that transmucosal transport of the P-gp substrates is strongly improved in the presence of thiolated chitosan. Glutathione and thiolated chitosan inhibit multidrug resistance P-glycoprotein activity in excised small intestine [46]. Therefore, when P-gp is inhibited, the bioavailability of substrates such as docetaxel is increased. Thiolated chitosan nanoparticles when administered orally could enhance oral bioavailability of DTX instead of current regimen of chemotherapy (IV injection). In addition, it can be regarded as a superior system when compared to other strategies that use P-gp/P450 inhibitors like cyclosporine-A with many side effects $[11,12]$.

\section{Conclusion}

In this study a core shell nanoparticulate system for the oral delivery of DTX with mucoadhesive properties for enhancing oral absorption of anticancer drugs is reported. Nanoparticles prepared in this study are superior to other nanoparticles such as PLGA NPs in terms of the following: (1) mucoadhesion property of thiolated chitosan provides better residence time of NPs in gastrointestinal tract, (2) achieving high drug entrapment efficiency, (3) surface hydrophilicity of chitosan NPs is favored compared to hydrophobic PLGA NPs, and (4) no hazardous organic solvent is used for the preparation of chitosan nanoparticles. Permeation study showed that nanoparticles could open tight junction of monolayer Caco- 2 cells and increase paracellular transportation. In vivo experiment with Wistar rats showed a significant increase in the half-life of DTX in plasma in comparison to that of F-DTX after IV injection. One dose of oral nanoparticle formulation can release DTX as sustainable manner for $216 \mathrm{~h}$ in comparison of $24 \mathrm{~h}$ for oral administration of F-DTX at the same dose of $10 \mathrm{mg} / \mathrm{kg}$ of DTX. The oral bioavailability of ChGSH-PMMA NPs was about 10-fold higher than that of oral F-DTX.

\section{Conflict of Interests}

The authors report no conflict of interests.

\section{Acknowledgments}

The authors would like to thank the Nanotechnology Research Centre of Tehran University of Medical Sciences for their support. The authors are also grateful to Mr. Khorasani for his kind assistance in in vivo experiments. 


\section{References}

[1] P. F. Escobar and P. G. Rose, "Docetaxel in ovarian cancer," Expert Opinion on Pharmacotherapy, vol. 6, no. 15, pp. 27192726, 2005.

[2] K. Iwao-Koizumi, R. Matoba, N. Ueno et al., "Prediction of docetaxel response in human breast cancer by gene expression profiling," Journal of Clinical Oncology, vol. 23, no. 3, pp. 422431, 2005.

[3] W. Eiermann, "Docetaxel-maximising outcomes towards cure in early breast cancer," Breast, vol. 15, supplement 3, pp. S13-S16, 2006.

[4] K. Gelmon, "The taxoids: paclitaxel and docetaxel," The Lancet, vol. 344, no. 8932, pp. 1267-1272, 1994.

[5] M. Aapro, "The scientific rationale for developing taxoids," AntiCancer Drugs, vol. 7, no. 2, pp. 33-36, 1996.

[6] S. Jones, "Head-to-head: docetaxel challenges paclitaxel," European Journal of Cancer, vol. 4, no. 4, pp. 4-8, 2006.

[7] I. E. L. M. Kuppens, T. M. Bosch, M. J. Van Maanen et al., "Oral bioavailability of docetaxel in combination with OC144-093 (ONT-093)," Cancer Chemotherapy and Pharmacology, vol. 55, no. 1, pp. 72-78, 2005.

[8] M. Shou, M. Martinet, K. R. Korzekwa, K. W. Krausz, F. J. Gonzalez, and H. V. Gelboin, "Role of human cytochrome P450 $3 \mathrm{~A} 4$ and $3 \mathrm{~A} 5$ in the metabolism of taxotere and its derivatives: enzyme specificity, interindividual distribution and metabolic contribution in human liver," Pharmacogenetics, vol. 8, no. 5, pp. 391-401, 1998.

[9] M. M. Malingré, J. H. Beijnen, and J. H. M. Schellens, "Oral delivery of taxanes," Investigational New Drugs, vol. 19, no. 2, pp. 155-162, 2001.

[10] L. Van Zuylen, J. Verweij, and A. Sparreboom, "Role of formulation vehicles in taxane pharmacology," Investigational New Drugs, vol. 19, no. 2, pp. 125-141, 2001.

[11] M. M. Malingré, D. J. Richel, J. H. Beijnen et al., "Coadministration of cyclosporine strongly enhances the oral bioavailability of docetaxel," Journal of Clinical Oncology, vol. 19, no. 4, pp. 11601166, 2001.

[12] M. Malingré, J. H. M. Schellens, O. Van Tellingen et al., "Metabolism and excretion of paclitaxel after oral administration in combination with cyclosporin A and after i.v. administration," Anti-Cancer Drugs, vol. 11, no. 10, pp. 813-820, 2000.

[13] A. Yousefi, F. Esmaeili, S. Rahimian, F. Atyabi, and R. Dinarvand, "Preparation and in vitro evaluation of a pegylated nanoliposomal formulation containing docetaxel," Scientia Pharmaceutica, vol. 77, no. 2, pp. 453-464, 2009.

[14] M. L. Immordino, P. Brusa, F. Rocco, S. Arpicco, M. Ceruti, and L. Cattel, "Preparation, characterization, cytotoxicity and pharmacokinetics of liposomes containing lipophilic gemcitabine prodrugs," Journal of Controlled Release, vol. 100, no. 3, pp. 331346, 2004.

[15] Y.-M. Yin, F. Cui, C. Mu et al., "Docetaxel microemulsion for enhanced oral bioavailability: preparation and in vitro and in vivo evaluation," Journal of Controlled Release, vol. 140, no. 2, pp. 86-94, 2009.

[16] K. Gao, J. Sun, K. Liu, X. Liu, and Z. He, "Preparation and characterization of a submicron lipid emulsion of docetaxel: submicron lipid emulsion of docetaxel," Drug Development and Industrial Pharmacy, vol. 34, no. 11, pp. 1227-1237, 2008.

[17] J. Kim, Y. Kim, S. Kim et al., "Hydrophobically modified glycol chitosan nanoparticles as carriers for paclitaxel," Journal of Controlled Release, vol. 111, no. 1-2, pp. 228-234, 2006.
[18] F. Esmaeili, R. Dinarvand, M. H. Ghahremani, S. N. Ostad, H. Esmaily, and F. Atyabi, "Cellular cytotoxicity and in-vivo biodistribution of docetaxel poly(lactide-co-glycolide) nanoparticles," Anti-Cancer Drugs, vol. 21, no. 1, pp. 43-52, 2010.

[19] Y. Dong and S. Feng, "Poly(D,L-lactide-co-glycolide)/montmorillonite nanoparticles for oral delivery of anticancer drugs," Biomaterials, vol. 26, no. 30, pp. 6068-6076, 2005.

[20] T. Nassar, S. Attili-Qadri, O. Harush-Frenkel et al., "High plasma levels and effective lymphatic uptake of docetaxel in an orally available nanotransporter formulation," Cancer Research, vol. 71, no. 8, pp. 3018-3028, 2011.

[21] H. Chen, Y. Zheng, G. Tian et al., "Oral delivery of DMABmodified docetaxel-loaded PLGA-TPGS nanoparticles for cancer chemotherapy," Nanoscale Research Letters, vol. 6, no. 1, pp. $1-10,2011$.

[22] E. Lee, H. Kim, I. Lee, and S. Jon, "In vivo antitumor effects of chitosan-conjugated docetaxel after oral administration," Journal of Controlled Release, vol. 140, no. 2, pp. 79-85, 2009.

[23] F. Esmaeili, R. Dinarvand, M. H. Ghahremani et al., "Docetaxelalbumin conjugates: preparation, in vitro evaluation and biodistribution studies," Journal of Pharmaceutical Sciences, vol. 98, no. 8, pp. 2718-2730, 2009.

[24] A. T. Florence, A. M. Hillery, N. Hussain, and P. U. Jani, "Nanoparticles as carriers for oral peptide absorption: studies on particle uptake and fate," Journal of Controlled Release, vol. 36, no. 1-2, pp. 39-46, 1995.

[25] Y. H. Lin, C. Chen, H. Liang et al., "Novel nanoparticles for oral insulin delivery via the paracellular pathway," Nanotechnology, vol. 18, no. 10, Article ID 105102, 2007.

[26] S. Peltier, J. Oger, F. Lagarce, W. Couet, and J. Benoit, "Enhanced oral paclitaxel bioavailability after administration of paclitaxelloaded lipid nanocapsules," Pharmaceutical Research, vol. 23, no. 6, pp. 1243-1250, 2006.

[27] M. Agüeros, V. Zabaleta, S. Espuelas, M. A. Campanero, and J. M. Irache, "Increased oral bioavailability of paclitaxel by its encapsulation through complex formation with cyclodextrins in poly(anhydride) nanoparticles," Journal of Controlled Release, vol. 145, no. 1, pp. 2-8, 2010.

[28] M. Roldo, M. Hornof, P. Caliceti, and A. Bernkop-Schnürch, "Mucoadhesive thiolated chitosans as platforms for oral controlled drug delivery: synthesis and in vitro evaluation," European Journal of Pharmaceutics and Biopharmaceutics, vol. 57, no. 1, pp. 115-121, 2004.

[29] V. M. Leitner, D. Guggi, A. H. Krauland, and A. BernkopSchnärch, "Nasal delivery of human growth hormone: in vitro and in vivo evaluation of a thiomer/glutathione microparticulate delivery system," Journal of Controlled Release, vol. 100, no. 1, pp. 87-95, 2004.

[30] F. Atyabi, F. A. Moghaddam, R. Dinarvand, M. J. ZohuriaanMehr, and G. Ponchel, "Thiolated chitosan coated poly hydroxyethyl methacrylate nanoparticles: synthesis and characterization," Carbohydrate Polymers, vol. 74, no. 1, pp. 59-67, 2008.

[31] J. Hombach, H. Hoyer, and A. Bernkop-Schnürch, “Thiolated chitosans: development and in vitro evaluation of an oral tobramycin sulphate delivery system," European Journal of Pharmaceutical Sciences, vol. 33, no. 1, pp. 1-8, 2008.

[32] F. A. Moghaddam, F. Atyabi, and R. Dinarvand, "Preparation and in vitro evaluation of mucoadhesion and permeation enhancement of thiolated chitosan-pHEMA core-shell nanoparticles," Nanomedicine, vol. 5, no. 2, pp. 208-215, 2009.

[33] F. Atyabi, F. Talaie, and R. Dinarvand, "Thiolated Chitosan nanoparticles as an oral delivery system for amikacin: in 
vitro and ex vivo evaluations," Journal of Nanoscience and Nanotechnology, vol. 9, no. 8, pp. 4593-4603, 2009.

[34] S. Saremi, F. Atyabi, S. P. Akhlaghi, S. N. Ostad, and R. Dinarvand, "Thiolated chitosan nanoparticles for enhancing oral absorption of docetaxel: preparation, in vitro and ex vivo evaluation," International Journal of Nanomedicine, vol. 6, no. 1, pp. 119-128, 2011.

[35] S. P. Akhlaghi, S. Saremi, S. N. Ostad, R. Dinarvand, and F. Atyabi, "Discriminated effects of thiolated chitosan-coated pMMA paclitaxel-loaded nanoparticles on different normal and cancer cell lines," Nanomedicine, vol. 6, no. 5, pp. 689-697, 2010.

[36] S. Saremi, F. Atyabi, S. P. Akhlaghi, S. N. Ostad, and R. Dinarvand, "Thiolated chitosan nanoparticles for enhancing oral absorption of docetaxel: preparation, in vitro and ex vivo evaluation," International Journal of Nanomedicine, vol. 6, no. 1, pp. 119-128, 2011.

[37] C. Chauvierre, D. Labarre, P. Couvreur, and C. Vauthier, "Radical emulsion polymerization of alkylcyanoacrylates initiated by the redox system dextran-cerium(IV) under acidic aqueous conditions," Macromolecules, vol. 36, no. 16, pp. 6018-6027, 2003.

[38] M. A. Arangoa, G. Ponchel, A. M. Orecchioni, M. J. Renedo, D. Duchêne, and J. M. Irache, "Bioadhesive potential of gliadin nanoparticulate systems," European Journal of Pharmaceutical Sciences, vol. 11, no. 4, pp. 333-341, 2000.

[39] A. Bernkop-Schnürch, M. Hornof, and T. Zoidl, "Thiolated polymers-thiomers: synthesis and in vitro evaluation of chitosan-2-iminothiolane conjugates," International Journal of Pharmaceutics, vol. 260, no. 2, pp. 229-237, 2003.

[40] A. Sadeghi, F. A. Dorkoosh, M. R. Avadi et al., "Permeation enhancer effect of chitosan and chitosan derivatives: comparison of formulations as soluble polymers and nanoparticulate systems on insulin absorption in Caco-2 cells," European Journal of Pharmaceutics and Biopharmaceutics, vol. 70, no. 1, pp. 270$278,2008$.

[41] I. Bravo-Osuna, G. Ponchel, and C. Vauthier, "Tuning of shell and core characteristics of chitosan-decorated acrylic nanoparticles," European Journal of Pharmaceutical Sciences, vol. 30, no. 2, pp. 143-154, 2007.

[42] F. Chen, Z. Zhang, F. Yuan, X. Qin, M. Wang, and Y. Huang, "In vitro and in vivo study of $\mathrm{N}$-trimethyl chitosan nanoparticles for oral protein delivery," International Journal of Pharmaceutics, vol. 349, no. 1-2, pp. 226-233, 2008.

[43] Z. Ma and L. Lim, "Uptake of Chitosan and associated insulin in caco- 2 cell monolayers: a comparison between Chitosan molecules and Chitosan nanoparticles," Pharmaceutical Research, vol. 20, no. 11, pp. 1812-1819, 2003.

[44] P. Artursson, T. Lindmark, S. S. Davis, and L. Illum, "Effect of chitosan on the permeability of monolayers of intestinal epithelial cells (Caco-2)," Pharmaceutical Research, vol. 11, no. 9, pp. 1358-1361, 1994.

[45] J. Smith, E. Wood, and M. Dornish, "Effect of Chitosan on epithelial cell tight junctions," Pharmaceutical Research, vol. 21, no. 1, pp. 43-49, 2004.

[46] E. K. Anderberg, C. Nystrom, and P. Artursson, "Epithelial transport of drugs in cell culture. VII: effects of pharmaceutical surfactant excipients and bile acids on transepithelial permeability in monolayers of human intestinal epithelial (Caco-2) cells," Journal of Pharmaceutical Sciences, vol. 81, no. 9, pp. 879887, 1992.
[47] Y. Dong and S. Feng, "Poly(D,L-lactide-co-glycolide)/montmorillonite nanoparticles for oral delivery of anticancer drugs," Biomaterials, vol. 26, no. 30, pp. 6068-6076, 2005. 

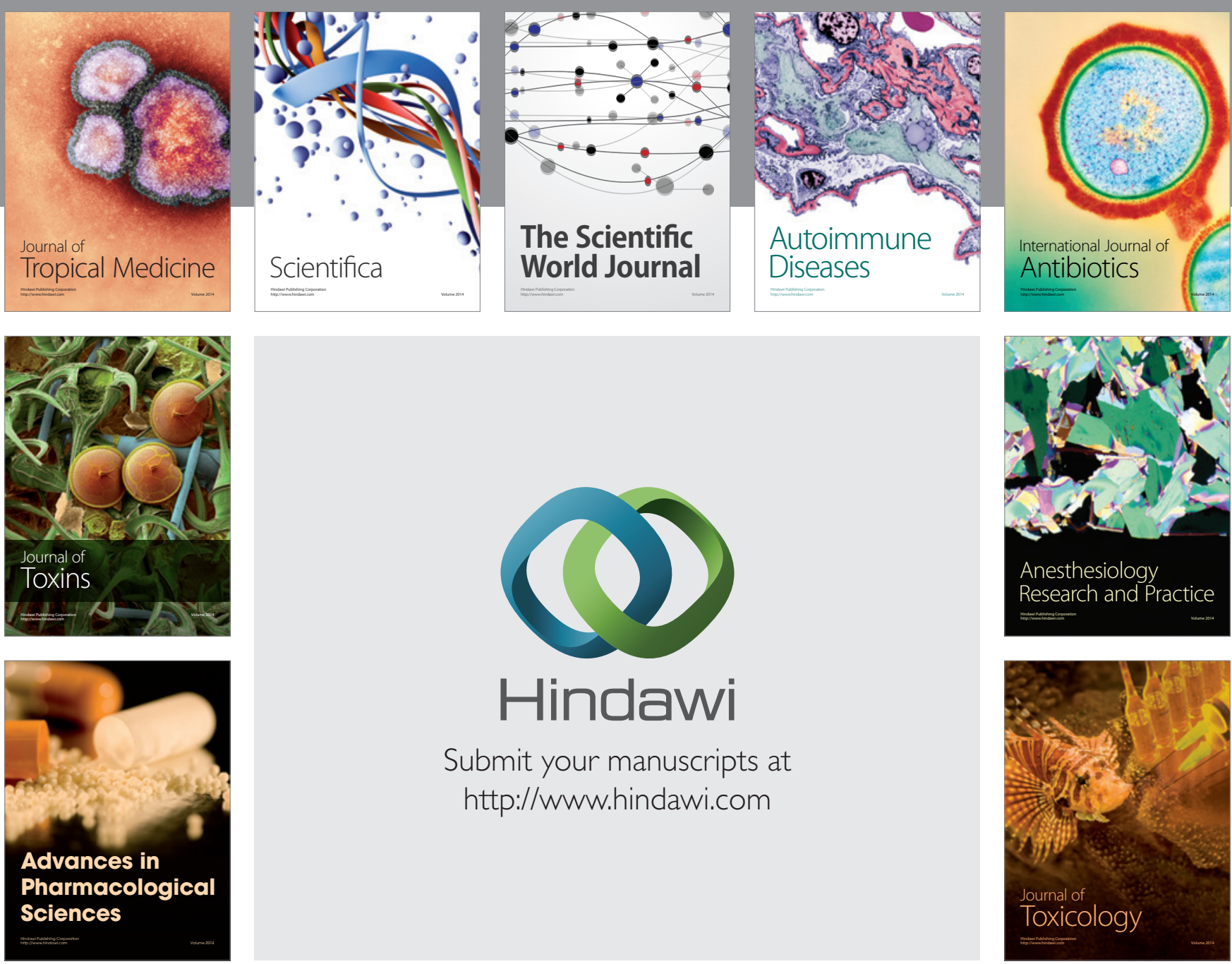

\section{Hindawi}

Submit your manuscripts at

http://www.hindawi.com
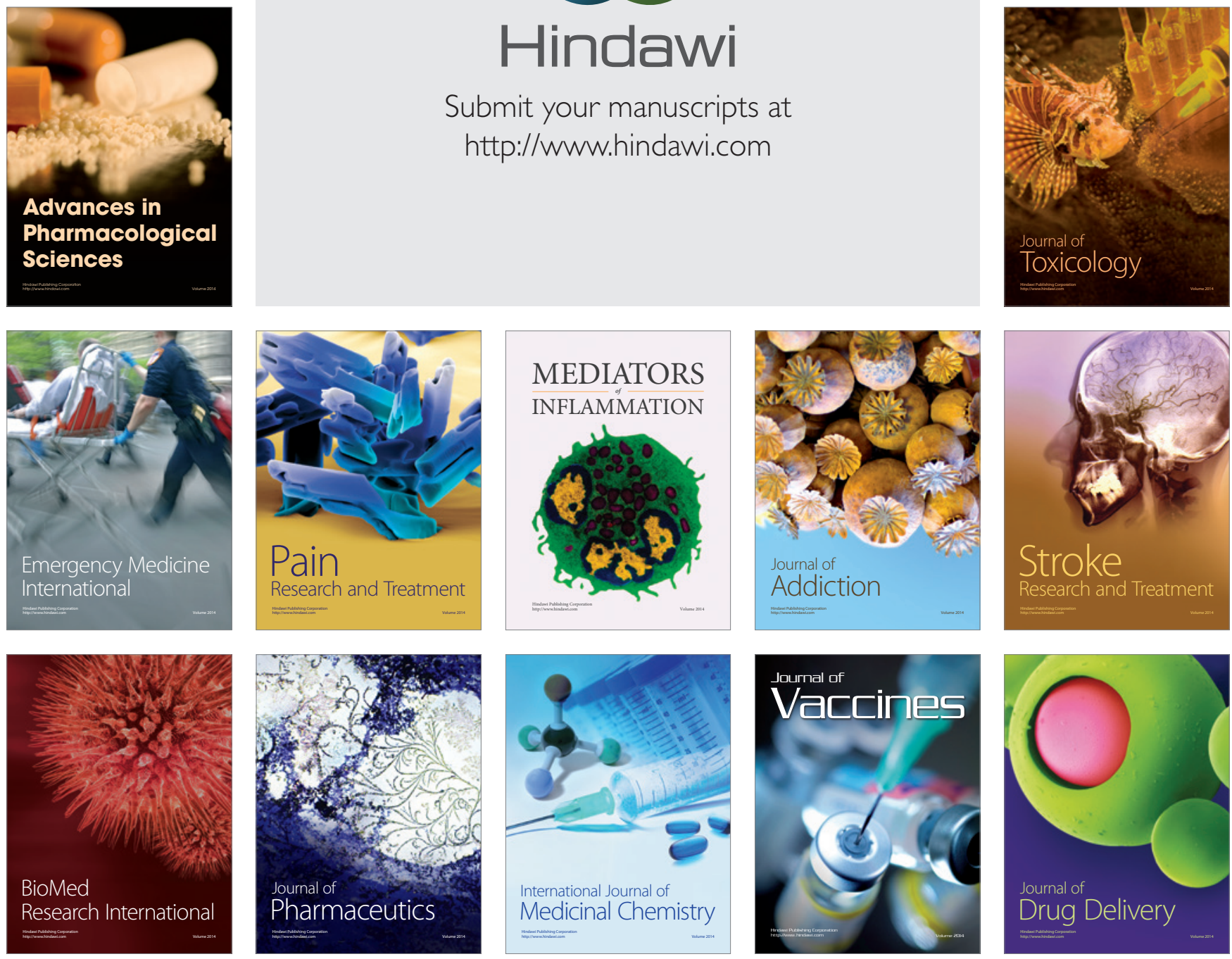